(Aus der Kgl. Universitäts-Frauenklinik Tübingen. Director: Prof. Dr. Döderlein.)

\title{
Der Einfluss der Scheidendesinfection auf die Morbidität im Wochenbett.
}

\author{
Von \\ Privatdocent Dr. K. Baisch.
}

Die Thatsache, dass auch überall da, wo die subjective geburtshilfliche Asepsis in der rigorosesten Weise durchgeführt wird, dennoch Fälle von Puerperalfieber nicht völlig verschwinden wollen, scheint immer und immer wieder denen Recht zu geben, die die Lehre von der Selbstinfection, d. h. der spontanen Infection der Kreissenden mit pathogenen, von ihrem eigenen Körper stammenden Keimen vertreten. Die logische Consequenz dieser Anschauung war die Forderung, nicht nur die Hände der geburtshilflichen Personen, sondern auch die äusseren Genitalien und die Scheide der Gebärenden vor und während der Geburt zu desinficiren. War die Hypothese von der Selbstinfection richtig, so musste mit der Entfernung und Vernichtung der Vulvar- und Scheidenkeime die Zahl der Fieberfälle im Wochenbett sich verringern.

Die Statistiken, die über die Erfolge präliminarer Scheidenspülungen aus den verschiedenen Kliniken publicirt worden sind, lauten nun aber sehr widersprechend. Auf der einen Seite stehen Ahlfeld und Hofmeier, von denen letzterer seinem Scheidendesinfectionsverfahren nachrühmt, dass er gerade ihm seine im Vergleich zu andern geburtshilflichen Kliniken so viel besseren Resultate verdanke; von den Gegnern hat besonders Menge erst neuerdings in sehr eindrucksvoller Weise seine Ansicht dahin ausgesprochen, dass die Antisepsis den Gebärenden keine Vortheile, sondern nur Nachtheile gebracht habe. 
Von den zahlreichen statistischen Zusammenstellungen über den Verlauf des Wochenbetts bej Gespülten und nicht Gespülten sind vor allem diejenigen von Interesse, die aus einer und derselben Klinik stammend über die Morbidität vor und nach Einführung der Scheidendesinfection berichten. Den Vergleich zwischen zwei verschiedenen Kliniken, von denen die eine spült, die andere nicht, kommt nach übereinstimmendem Urtheil keinerlei Beweiskraft zu, denn ausser dem Vorhandensein oder Fehlen von Scheidenkeimen wirken zweifellos noch eine grosse Zahl von localen Factoren auf die Entstehung von Puerperalfieber ein. Die Unzulänglichkeit solcher Vergleiche wird am besten illustrirt durch die Thatsache, dass gerade in den Kliniken der beiden überzeugtesten Anhänger der Scheidendesinfection die grössten Unterschiede herrschen: A hlfeld hat eine puerperale Morbidität von $35 \mathrm{pCt}$., Hofmeier eine solche von nur $9,5 \mathrm{pCt}$. Dieselbe niedrige Morbidität aber wie Hofmeier haben auch ohne Spülungen Mermann, Leopold, v. Rosthorn und viele Andere.

Aber auch wo die Ergebnisse an einer und derselben Klinik vor und nach Einführung der Spülungen verglichen werden, ergaben sich merkwürdiger Weise sehr verschiedene Resultate. Steffeck constatirte nach Einführung der Scheidendesinfection eine Abnahme der Morbidität von 19,4 auf 5,9 und 7,5 pCt., Ahlfeld von 62 auf 45 pCt., Holowko in Dorpat von 16 auf 4 pCt., Günther von 47 auf 30 pCt., Frommel von 11 auf 5-7 pCt.

In andern Kliniken wieder war es gerade umgekehrt. Fehling fand nach Aussetzen der Spülungen einen Rückgang der Morbidität und zwar in Basel von 35 auf 9 pCt., in Halle ron 27 auf 13 pCt. Heinricius hatte bei Spülung 16, ohne sie 11 pCt. Morbidität. v. Rosthorn konnte einen Unterschied überhaupt nicht constatiren, er hatte das eine Mal 10,3 pCt., das andere Mal 10,6 pCt. Erkrankungen. Die grössten Versuchsreihen publicirten Leopold und Goldberg. Sie beobachteten bei 3400 Gespülten und nicht Operirten eine Morbidität von 1,47 pCt., bei 1369 Niehtgespülten eine solche von $0,9 \mathrm{pCt}$.

Um auch den Einwand zu entkräften, dass innerhalb derselben Klinik zu verschiedenen Zeiten verschiedene Einflüsse auf die Höhe der Infection sich geltend machen und dadurch die Resultate trüben könnten, stellte Krönig die Versuche in der Weise an, dass abwechselnd je eine Kreissende vor der Geburt gespült wurde, die andere nicht. Von 465 Nichtausgespülten erkrankten mit Fieber 
über $38,5^{0}$ im Rectum 36 pCt., von 515 Ausgespülten dagegen 49 pCt. In beiden Serien starb je eine Wöchnerin an Sepsis. Vergleicht man nur diejenigen Kreissenden, die während der Geburt auch innerlich untersucht wurden, so fieberten von 123 Gespülten 44 pCt., von 78 Nichtgespülten jedoch nur 20,5 pCt. Diese Versuchsanordnung entschied also sehr zu Ungunsten der Desinficirten and Bretschneider, der diese Versuche Krönigs weiterführte, kam im wesentlichen $\mathrm{zu}$ denselben Resultaten.

Hatte Steffeck gegen die Resultate Leopold's eingewandt, dass in Dresden die Desinfection vielleicht nicht gründlich genug vorgenommen werde und verlangt, dass nicht nur die Scheide, sondern auch der Cervicalkanal alle 2 Stunden mit Sublimat ausgerieben werde, so führte nun Sticher die Resultate Krönig's auf eine zu energische Desinfection zurück und wollte die Scheide nicht mit Sublimat ausgerieben, sondern nur mit Lysol ausgespült wissen.

So steht Statistik gegen Statistik, Behauptung gegen Behauptung und die ganze wichtige und praktisch einschneidende Frage scheint auf einem todten Punkt angelangt zu sein.

Thatsächlich kommt auch allen bisher angestellten statistischen Erhebungen keine genügende Beweiskraft zu. Die Schuld daran ist in dem Umstand zu suchen, dass es bei so complicirten Lebensprocessen, wie es die Entstehung des Puerperalfiebers ist, ausserordentlich schwer hält, alle Faktoren mit Ausnahme des einen, dessen Wirkung untersucht werden soll, in beiden Parallelreihen völlig gleich zu gestalten. Da dies bei keiner der bisherigen Versuchsanordnungen gelungen ist, darf es uns nicht wundern, wenn weder für die Frage der Zweckmässigkeit der Scheidenspülungen als Prophylacticum gegen Wochenbettfieber noch für das Problem der Selbstinfection allgemein anerkannte Antworten sich ergeben haben.

Es sind 2 principielle Einwendungen, die gegen die Art der bisherigen Beweisführung und Statistik erhoben werden müssen.

Wenn wir den Einfluss der Scheidenkeime auf Entstehung und Häufigkeit von Puerperalfieber dadurch kennen lernen wollen, dass wir die Geburten abwechselnd bei keimhaltiger und bei keimarmer Vagina verlaufen lassen, so vermögen wir aus dem Verlauf der Wochenbetten nur dann sichere Schlüsse abzuleiten, wenn wir alle anderen Infectionsquellen ausgeschlossen oder auf beiden Seiten völlig identisch gestaltet haben. Die Hauptquelle der Infectionen 
ist nun zweifellos die touchirende, durch keine Desinfectionsmethode absolut keimfrei zu machende Hand. In den bisherigen Versuchsanordnungen ist keinerlei Garantie gegeben; weder für völligen Ausschluss, noch für gleichmässige Vertheilung der Gefahr der Handinfection auf beide Gruppen von Kreissenden. Gerade bei den präliminaren Scheidenspülungen ist die Gefahr der Uebertragung von Handkeimen eine besonders grosse. Wie will man den Einwand widerlegen, dass beim Ausreiben der Scheide und Cervix während des Durchfliessens von 1 Liter Spülflüssigkeit nicht Handkeime direct in die weiche Cervical- und Scheidenschleimhaut eingeimpft werden? Treten nun bei den Desinficirten mehr Fieberfälle auf, als bei Nichtdesinficirten, so kann dies die Ursache in einer durch die Spülung erhöhten Uebertragung der Handkeime haben. Treten weniger Fälle von Fieber anf, so wissen wir nicht, ob der Grund in der Vernichtung der Scheidenkeime oder in der Entfernung eingeschleppter Handkeime zu suchen ist. Da es zudem nach den bakteriologischen Untersuchungen von Döderlein und Steffeck nicht gelingt, durch einmalige, ja selbst nicht durch wiederbolte Spülungen die Vaginalbakterien für die Dauer der Geburt zu entfernen, so ist auch bei Gespülten die. Möglichkeit einer Erkrankung durch Scheidenkeime vielleicht nur herabgesetzt, jedenfalls nicht ausgeschlossen.

Beide Infectionsgelegenheiten, die durch Händekeimübertragung; wie die durch Scheidenbakterien sind also bei Gespülten wie bei Nichtgespülten gegeben und wir vermögen in keiner Weise zu sagen, in welchem Grade durch die Spülungen eine von beiden und welche beeinflusst wird.

Die Versuche, wie sie bisher angestellt wurden, könnten daher höchstens die Frage lösen, ob Scheidenspülungen mit antiseptischen Flüssigkeiten. während der Geburt geeignet sind, die Wochenbettsmortalität herabzusetzen. Ob sie dies auf dem Wege der Vernichtung von bei der Untersuchung eingeschleppten Händekeimen oder durch Entfernung und Abtödtung der präexistirenden Scheidenbakterien bewirken, müsste dabei völlig dahin gestellt bleiben. In der That suchen die Vertheidiger der Scheidendesinfection den von ihnen behaupteten Nutzen der Spülungen bald aus der einen, bald aus der anderen Wirkungsweise abzuleiten.

Der zweite Einwand, der gegen den Werth der bisherigen Statistik erhoben werden muss, richtet sich gegen Begriff und 
Definition von Puerperalfieber. Es ist in den publicirten Statistiken entweder überhaupt jeder Fall von Fieber im Wochenbett bei Berechnung der Wochenbettsmorbidität mitgezählt, also auch Fälle von Mastitis, Influenza etc., die mit Vornahme oder Unterlassung von Scheidenspülangen ätiologisch überhaupt nicht zusammenhängen. Oder die Abtrennung ist nach rein klinischen Ueberlegungen ausgeführt. Wie unzuverlässig aber eine derartige Ausscheidung ist, erhellt deutlich aus der von Döderlejn hervorgehobenen Thatsache, dass die Häufigkeit dieser accidentellen Erkrankungen bei Stellung der Diagnose lediglich nach klinischen Gesichtspunkten bei den einzelnen Autoren zwischen 2 und $36 \mathrm{pCt}$. schwankt. Es ist einleuchtend, dass bei solcher Unsicherheit der Diagnose alle Berechnungen über den Einfluss dieser oder jener prophylaktischen oder therapeutischen Maassnahmen auf die Höhe des. Puerperalfiebers nur approximativen Werth beanspruchen können. Auch die Annahme, dass bei Gegenüberstellung Desinficirter und Nichtdesinficirter die Fehler in der Abscheidung der extragenitalen Fieberfälle sich gegenseitig aufheben, wäre nur beim Vergleich sehr grosser Zahlen einigermaassen gerechtfertigt. Ganz unmöglich aber ist es, die Berechnung der absoluten Häufigkeit der rein genitalen Erkrankungen im Wochenbett und gar nun diejenige der Selbstinfectionen vorzunehmen, so lange die diagnostischen Hilfsmittel, nach denen die Scheidung vorgenommen wird, die Abtrennung innerhalb der weitesten Grenzen erlauben. Wohl versichert jeder Autor, dass bei dieser Abtrennung mit der schärfsten Kritik vorgegangen werden müsse - ohne einen objectiven Maassstab schützt aber der gute Wille allein nicht vor den schwersten diagnostischen Täuschungen.

Will man das Problem der Selbstinfection und der Bedeutung der Scheidendesinfection wirklich fördern, so genügt übrigens die Trennung der Fieberfälle nach genitalen und accidentellen Erkrankungen noch bei Weitem nicht. Auch die Fieberfälle, die sicher genitalen Ursprungs sind, sind selbst wieder unter sich so verschieden, dass wir aus ihrer blossen Addition nie einen Einblick in ihre ätiologische Natur gewinnen können, die doch in erster Linie von Interesse ist. $O b$ in diesen Fällen das Fieber durch Keimascendenz bei Eihautretention, ob durch Gonokokken, ob durch Streptokokken hervorgerufen wird, bedingt erst die wesentlichen Unterschiede in der Auffassung der Aetiologie, der Therapie und der Prophylaxe. Nirgends aber in den bisher mitgetheilten 
Statistiken über den Einfluss der Scheidendesinfektion finden wir diese Fragen berücksichtigt und wir wissen nicht, ob bei Gespülten eine Abnahme oder Zunahme der Fieberfälle auf Rechnung der accidentellen Erkrankungen oder der Ascendenz der Gonorrhoe oder saprophytischer Keime oder der specifischen puerperalen Infectionserreger, der Streptokokken zu setzen ist.

Auch von diesem Gesichtspunkt aus erfahren wir also bei einer nach der bisherigen Methode aufgestellten Statistik der Spülungen bei der Geburt höchstens die allgemeine Thatsache, dass bei ihrer Anwendung häufiger oder seltener Fieber im Wochenbett auftritt. Ueber den causalen Zusammenhang erfahren wir nichts.

$\mathrm{Zu}$ alledem kommt noch ein weiterer Punkt, der auch den Werth dieses einzigen Resultates wesentlich herabmindert. Die Wirkung der Scheidendesinfection ist keine einfache, sondern sie setzt sich selbst wieder aus mehreren Componenten zusammen. Neben ihrer baktericiden Eigenschaft kommt der Spülung noch eine physikalisch-chemische Wirkung zu. Sie entfernt den Vaginalschleim, tödtet und lockert die obersten Schichten des Cervixund Scheidenepithels, ändert die saure Reaction des Scheidensecretes. Mit der Entkeimung verbindet sich eine Alteration der physiologischen Verhältnisse der Vagina, deren Wirkung nicht von vornherein und ohne Weiteres als eine im gleichen Sinne sich vollziehende bezeichnet werden kann. Gerade auf diese mechanischen und physikalisch-chemischen Wirkungen legten Baumm und Menge bei der Verwerfung der Scheidenantisepsis ganz besonderes Gewicht. Zudem bestehen hier zweifellos Unterschiede zwischen den verschiedenen Desinficientien und den verschiedenen Methoden ihrer Anwendung: Saure Antiseptica werden andere Wirkungen entfalten als neutrale und alkalische, schleimlösende andere als solche, die das Mucin und Eiweiss nicht verändern etc.

So wäre es wohl denkbar, dass ein Desinfectionsmittel, was es auf der einen Seite durch Abtödtung pathogener Keime nützt, auf der anderen durch Schädigung der vitalen Eigenschaften wieder verdirbt und ein statistisch sich ergebender Nachtheil der Spülungen brauchte daher noch nicht ohne Weiteres auf einen Nachtheil der Desinfection überhaupt schliessen zu lassen, sondern könnte auch lediglich nur auf eine falsche Anwendung und Ausführung des an sich richtigen Princips zurückzuführen sein.

Alle diese Momente wirken zusammen, um die Beweiskraft der statistischen Ergebnisse der Scheidendesinfection, so wie sie mit 
den bisherigen Methoden ermittelt wurden, stark zu beeinträchtigen und die diametral sich gegenüberstehenden Resultate der Autoren erklärlich erscheinen zu lassen.

Es frägt sich, ob diese Mängel der Methoden beseitigt werden können.

Döderlein hat in seinem Vortrag über Entstehung und Verhütung des Kindbettfiebers auf dem X. Gynäkologen-Congress in Würzburg die Forderungen formulirt, die an eine Wochenbettstatistik gestellt werden müssen, wenn sie zu wissenschaftlichen Zweeken verwendbar sein soll.

Was zunächst den ersten Einwand gegen die bisherigen Statistiken anlangt, dass bei der.Unmöglichkeit, die Hand völlig keimfrei zu machen, bei eingetretener Infection deren Quelle, ob Scheide, ob Hand, nicht mehr zu ermitteln ist, so wird er durch Verwendung der Gummihandschuhe und nur durch sie mit einem Schlage beseitjgt. Mit ihnen wird die Handinfection ausgeschaltet. Da wo auch jede Manipulation an den äusseren Genitalien der Kreissenden: Reinigung, Desinfection, Dammschutz, Katheterismus u. s. w. nur mit handschuhbekleideter Hand vorgenommen wird, ist in Fällen von Fieber im Wochenbett mit einer an Gewissheit grenzenden Wahrscheinlichkeit die Ursache anderswo als in den Händekeimen der geburtshilflichen Personen za suchen.

Man hat gegen den Werth der Handschuhe eingewendet, dass in den verschiedenen Kliniken, in denen sie in Anwendung kamen, der danach eingetretene Rückgang der Morbidität nur unbedeutend sei. Immerhin war überall ein solcher Rückgang zu constatiren. Er betrug in Breslau 2,3 pCt., in Strassburg 2,7 pCt., in Graz sogar 10,4 pCt. Die Mögliehkeit, mit irgend einem der modernen Händedesinfectionsverfahren die Hand frei von septischen Stoffen zu machen, ist bei der allgemein eingehaltenen Abstinenz und Noninfection geburtshilflicher Personen in Kliniken und Gebäranstalten doch eine so weitgehende, dass gar nicht zu erwarten ist, dass bei Verwendung von Handschuhen die Morbidität sehr stark sinken würde. Das ändert aber nichts an der Thatsache, dass wir nur mit ihrer Hilfe im Stande sind, die Händeinfection mit derjenigen Sicherheit zu eliminiren, die bei Verwerthung der Resultate zu wissenschaftlichen Zwecken unerlässlich ist.

Natürlich wird man bei der Ermittlung der Scheidendesinfektionswirkung die durch Hautkeime verschuldeten Fieberfälle weiterhin noch dadurch ausschliessen, dass man nur solche Wöchnerinnen 
332 Baisch, Der Einfluss der Scheidendesinfection auf die Morbidität etc.

zum Vergleich heranzieht, die schon als Schwangere einige Zeit in der Klinik wohnten und alle die nicht für die Statistik verwerthet, die kreissend eingetreten sind, da bei ihnen ausserhalb der Klinik durch Untersuchung von Seiten der Hebammen etc. bereits Infektionen erfolgt sein können. Ebenso müssen ausgeschlossen werden alle operativen Entbindungen, da hier durch Verletzungen, Quetschungen, Blutverluste, lange Geburtsdauer etc. complicirte Verhältnisse gegeben sind, die die Beurtheilung des Causalzusammenhanges erschweren. Der Einwand, den Sticher gegen Krönig erhebt, der ebenfalls die operativ Entbundenen nicht berücksichtigt, dass dadurch eine Auslese besonders günstiger Fälle getroffen und ein Urtheil über die Leistungsfähigkeit des Verfahrens unmöglich gemacht werde, ist ganz und gar nicht zutreffend. Es handelt sich darum, die Beurtheilung durch möglichst einfache Gestaltung der Verhältnisse zu erleichtern, um die Wirkung eines einzelnen Factors, der Spülungen klar hervortreten zu Jassen. Die Frage der Zweckmässigkeit der Scheidendesinfection bei operativen Geburten muss für sich gelöst werden.

Selbst durch Abtrennung aber aller dieser "unreinen" Fälle der kreissend Eingetretenen und der operativ Entbundenen - ist natürlich die Möglichkeit der Uebertragung von Aussenkeimen immer noch nicht völlig beseitigt. Döderlein hat in jenem Vortrag bereits darauf hingewiesen und in der Diskussion wurden auch von anderer Seite, inbesondere von Bumm sehr instructive Belege dafür beigebracht, dass uncontrollirbare Vorgänge z. B. heimliches Selbsttouchiren und Anderes mehr Infectionen vermitteln können, die wir nicht zu verhüten im Stande sind. Die Zahl solcherInfectionen wird aber immerhin eine kleine und ihre Bedeutung für das Gesammtresultat um so geringer sein, je grösser die Zahl der Wöchnerinnen ist, die wir unseren Vergleichen and Berechnungen zu Grunde legen.

Der trotz systematischer Verwendung von Gummihandschuhen noch verbleibende Rest von Fieberfällen enthält nun - und damit kommen wir zum zweiten der erhobenen Einwände - vor Allem noch die Gesammtheit der extragenitalen Erkrankungen. Sie in zuverlässiger, objektiver Weise abzuscheiden, ist ein unerlässliches Postulat bei Erforschung des Puerperalfiebers. Die einzige Möglichkeit dazu bietet die bakteriologische Untersuchung der Uteruslochien, wie sie längst von Döderlein, Bumm and Krönig gefordert wird. Sie wird in Tübingen in jedem einzelnen 
Fall von Fieber im Wochenbett ausgeführt, sobald die Temperatur über $38^{\circ} \mathrm{im}$ Rectum steigt, selbst wenn - z. B. bei abscedirender Mastitis - alle Wahrseheinlichkeit dafür spricht, dass die Quelle des Fiebers nicht im Uterus zu suchen ist.

Es sind gegen diese Methode und die Berechtigung, aus dem Befund von pathogenen Keimen Schlüsse auf die Ursache des Fiebers zu ziehen, von verschiedenen Seiten Einwände erhoben worden. Sie gründen sich auf die Thatsache, dass fast von allen Untersuchern der Uteruslochien: Döderlein, Winternitz, Stähler-Winkler, Wormser, Stoltz, Schauenstein u. A. auch aus dem Uterus normaler Wöchnerinnen zuweilen Streptokokken gezüchtet werden konnten. Das setzt jedoch für die uns hier beschäftigende Frage die Brauchbarkeit der Methode nicht herab. Es handelt sich für uns nur darum, bei Fiebernden die Ursache der Temperaturerhöhung zu ermitteln. Finden wir bei Fiebernden pathogene Keime im Uterus und nirgends am übrigen Körper der Wöchnerin einen Krankheitsherd, so wäre es gewiss doctrinär, die causale Bedeutung des positiven Befundes im Uterus für das vorhandene Fieber deswegen leugnen zu wollen, weil auch bei Nichtfiebernden zuw.eilen Streptokokken in den Uteruslochien gefunden werden. Auch halten wir uns nicht für berechtigt, eine genitale Fieberursache auszuschliessen, wennn wir bei sonstigen localen Störungen einen positiven bakteriologischen Befund an den Uteruslochien erheben. Wohl aber glauben wir dies thun zu dürfen, wenn bei ausgesprochener extragenitaler Erkrankung: Angina, Mastitis, Pneumonie ete. die Uteruslochien bei ein- oder mehrmaliger Untersuchung sich als steril erwiesen haben.

Es kann bei diesem Vorgehen nur nach der Richtung hin ein Irrthum unterlaufen, dass der eine oder andere extragenitale Fieberfall zu Unrecht als puerperal gedeutet wird. Es ist dies zweifellos der geringere Fehler, denn es besteht im Allgemeinen eher die Neigung $\mathrm{zu}$ viel und nicht zu wenig accidentelle Erkrankungen auszuscheiden.

Ebenso werthvoll und ganz unbestreitbar ist aber der weitere Gewinn, den die bakteriologische Diagnostik mit sich bringt, die Möglichkeit mit ihrer Hilfe in jedem einzelnen Fall von Puerperalfieber die verursachenden Mikroben festzustellen und dadurch eine weitere Eintheilung der Erkrankungen auf ätiologischer Basis zu gewinnen.

Wir sind somit bei Verwendung von Gummihandschuhen in 
334 Baisch, Der Einfluss der Scheidendesinfection auf die Morbidität etc.

der Prophylaxe der Keimübertragung und bei Benutzung der bakteriologischen Diagnostik mit grosser Zuverlässigkeit in der Lage, die Häufigkeit derjenigen puerperalen Infeetionen festzustellen, die weder einer Keimeinschleppung noch einer accidentellen Erkrankung ihren Ursprung verdanken und wir vermögen weiter durch die Feststellung der fiebererregenden Mikroben unter gleichzeitiger Berücksichtigung der klinischen Erfahrungen werthvolle Aufschlüsse über das Zustandekommen der Infection (Gonorrhoe, saprische Keime bei Eihautretention u. s. w.) zu erhalten.

Es wäre von hervorragendem wissenschaftlichem wie praktischem Interesse, wenn eine grössere Anzahl von Kliniken unter den genannten Cautelen Resultate sammeln und publiciren würde. Vor Allem würden Untersuchungen über den Einfluss prophylaktischer Maassnahmen, wie sie z. B. die Scheidenspülungen darstellen, erst dadurch ein exaktes Fundament gewinnen und könnten in ihren Ergebnissen auf die überzeugende Kraft Anspruch erheben, die wir an den bisher mitgetheilten noch so sehr vermissen.

Die im Folgenden mitgetheilten' Untersuchungen über den Einfluss von Sublimat-Scheidenspülungen auf den Verlauf des Wochenbetts erfüllen nun die aufgestellten Bedingungen. Sämmtliche Kreissende wurden nur mit Gummihandschuhen untersucht und ebenso fand jede Berührung der Genitalien bei der Desinfection, beim Katheterisiren, beim Dammschutz nur mit gummibedeckten Händen statt. Die allgemeinen hygienischen Maassnahmen waren bei allen Kreissenden dieselben: bevor die Kreissende den Gebärsaal betritt, bekommt sie ein Vollbad und frische Wäsche. Vor der inneren Untersuchung werden die äusseren Genitalien nach Kurzschneiden der Schamhaare mit warmem Wasser und Seife 5 Min. lang gewaschen, darnach mit Spiritus und Sublimat, neuerdings mit Sublamin abgerieben. Diese Sublimatwaschung wird vor jeder inneren Untersuchung wiederholt.

Die Untersuchungen erstrecken sich auf die Jahre 1902-1905 und umfassen nunmehr 1000 spontan niedergekommene Hausschwangere. Die Zahl "der inneren Untersuchungen ist zum Theil eine recht beträchtliche, bis zu 30 bei einer Kreissenden, da ausser der Oberhebamme und dem Assistenzarzt der geburtshilflichen Abtheilung noch mehrere Volontäre, Hauspraktikanten und Studenten bei jeder Geburt zugegen sind.

Von den Kreissenden wurden 500 im Beginn der Geburt ausgespült, 500 nicht. Die Scheidenspülungen wurden mit je 1 Liter 
einer $1 \%$ Sublimatlösung vorgenommen und es möge hier noch ausdrücklich hervorgehoben werden, dass sich unsere Ergebnisse darnach nur auf die Wirkungen der Sublimatdesinfection der Scheide beziehen. Die Spülung wurde in der Weise ausgeführt, dass während des Durchfliessens der Lösung die Scheidenwände mit 2 Fingern der handschuhbekleideten Hand vorsichtig und schonend, aber doch so gründlich wie möglich ausgerieben wurden.

Die Wochenbetten unserer 1000 Spontangeburten verliefen nun folgendermaassen:

Von 500 Nichtgespülten fieberten insgesammt $40=8 \mathrm{pCt}$.

$$
\text { n } 500 \text { Gespülten } \quad \text { n } \quad 64=12,8 \mathrm{pCt} \text {. }
$$

Die Gespülten fieberten also in bedeutend grösserer Häufigkeit als die Ungespülten.

Von den Nichtgespülten wurden 127 bei der Geburt innerlich nicht untersucht.

Diese 127 überhaupt nicht Untersuchten hatten mit 11 Fieberfällen eine Morbidität von $8,6 \mathrm{pCt}$.

Vergleichen wir nur die innerlich Untersuchten in beiden Reihen - und die Gespülten sind eo ipso auch innerlich untersucht, so finden wir:

Von 373 Nichtgespülten fieberten insgesammt $29=7,7 \mathrm{pCt}$.

$$
\text { " } 500 \text { Gespülten " " } \quad 64=12,8 \text { pCt. }
$$

Das Verhältniss ist nahezu dasselbe und beweist, dass es völlig irrelevant ist, ob gar nicht oder ob mit handschuhbekleideter Hand untersucht wird. Die Handschuhe erfüllen demnach vollkommen ihren Zweck, die Uebertragung von Händekeimen auszuschliessen. Wir ersehen aber noch das Weitere aus den obigen Zahlen, dass auch die von Vielen gefürchtete Gefahr der Uebertragung von Vulvarkeimen auf die inneren Genitalien nicht besteht. Untersuchte und Nichtuntersuchte haben dieselbe Morbidität, rund 8 pCt., was nicht der Fall sein könnte, wenn der Transport von Vulvarkeimen bei der Untersuchung einen nennenswerthen Einfluss auf die puerperale Morbidität bei Spontarıgeburten besässe. Es berechtigt uns das, auch die Nichtuntersuchten mit den Untersuchten zusammenzunehmen und insgesammt den Gespülten gegenüberzustellen.

Es wäre denkbar, wenn auch bei der Zahl der Verglichenen nicht gerade sehr wahrscheinlich, dass durch eine zufällige hohe Betheiligung extragenitaler Fieberfälle auf Seiten der Ausgespülten 
die grosse Differenz sich erklären und die Gegenüberstellung somit nichts gegen den Werth der Spülungen beweisen würde.

Es sind im Ganzen 28 Fälle, in denen neben Sterilität der Uteruslorhien sich ein das Fieber erklärender Krankheitsherd am übrigen Körper der Wöchnerin fand. Die Fälle vertheilen sich gleichmässig auf die Gespülten und Nichtgespülten.

Bei den Gespülten fand sich als Fieberursache:

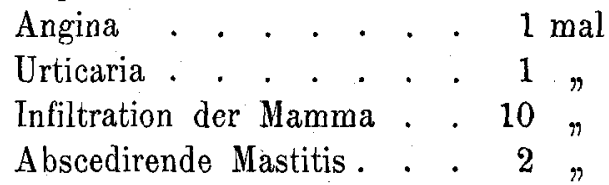

Bei den Nichtgespülten:

\begin{tabular}{|c|c|c|c|}
\hline Bronchitis & . & & \\
\hline Pleuritis. & ${ }^{\circ}$ & & \\
\hline Pyelonephritis & $\cdot$ & . & \\
\hline Erysipelas faciei & & & \\
\hline Infiltration der 1 & Mamma & . & \\
\hline Abscedirende $\mathrm{Ma}$ & astitis : & & \\
\hline
\end{tabular}

Die Häufigkeit der accidentellen Erkrankungen beträgt demnach in unserem Material 2,8 pCt. aller Wöchnerinnen und $27 \mathrm{pCt}$. aller Fiebernden.

Ziehen wir auf beiden Seiten diese extragenitalen Fieberfälle ab, so bleiben:

von 500 Nichtgespülten fieberten genital $26=5,2$ pCt.

"500 Gespülten " " $\quad 50=10,0$ "

und wenn wir die Nichtuntersuchten wieder bei Seite lassen:

von 373 Nichtgespülten fieberten genital $20=5,3$ pCt.

n500 Gespülten $" \quad 50=10,0$ "

Der Procentsatz der Fiebernden ist bei den Gespülten nahezu doppelt so hoch wie bei den Nichtgespülten und beachtenswerther Weise besteht auch hier kein Unterschied, ob die Nichttouchirten mitgerechnet oder weggelassen werden.

Die Erkrankungshöhe ist fast genau dieselbe, wie sie Döderlein für 1000 mit Handschuhen untersuchte, spontan niedergekommene Hausschwangere bis zum Jahre 1903 ermittelt hatte, die ebenfalls nicht prophylactisch desinficirt waren. Er fand 8,9 pCt. Gesammterkrankungen und 4,8 pCt. Genitalerkrankungen.

Der verschiedenen klinischen Dignität des Fiebers im Wochenbett, soweit es genitalen Ursprungs ist, trägt man wohl am 
besten Rechnung, wenn man die einzelnen Fälle nach der Dauer der Erkrankung abgrenzt und speciell die „Eintagsfieber" gesondert. werthet.

Bezeichnen wir als "gestört" diejenigen Wochenbetten, in denen nur eine einmalige Steigerung über $38,5^{\circ}$ Rectalmessung auftrat, als "krank" dagegen die, bei denen die Steigerung durch mehrere Tage anhielt oder sich wiederholte, so haben wir:

von 500 Nichtgespült. war.gestört $15=3,0 \mathrm{pCt}$., krank $11=2,2 \mathrm{pCt}$.

"500 Gespülten $\quad " \quad 32=6,4, \quad$ " $18=3,6$ "

Schwerere wie leichtere Störungen finden sich also bei den Gespülten etwa doppelt so häufig, wie bei den Nichtgespülten. Und berücksichtigen wir nur untersuchte Kreissende, so ergiebt sich nahezu dasselbe Bild:

von 373 Nichtgespült. waren gestört $12=3,2$ pCt., krank $8=2,1$ pCt. "500 Gespülten " " $32=6,4 \quad$ " $18=3,6$ "

Es sind somit weder die schwereren Wochenbettserkrankungen durch die Scheidendesinfection verringert worden, noch die leichteren Störungen. Man kann sich vielmehr dem Eindruck nicht verschliessen, dass beide durch sie erheblich, bis auf die doppelte Höhe gesteigert wurden.

Auch die Zeit des Fiebereintritts und die Höhe der Körperwärme wird man bei der Beurtheilung des klinischen Charakters der Infection nicht vernachlässigen dürfen.

Bei den Gespülten fällt für die Kranken wie für die Gestörten das Maximum der Fieberfälle auf den 4.-7. Tag, also noch in die erste Woche. Es fieberten erstmals

$$
\begin{aligned}
& \text { am 4. Tag } 9 \text { Pat. }=18 \text { pCt. } \\
& \begin{array}{rrrrr}
n & 5 . & n & 7 & =14 \\
n & 6 . & n & 10 & =20 \\
n & 7 . & n & 12 &
\end{array}
\end{aligned}
$$

Bei den Nichtgespülten dagegen lässt sich eine solch' ausgesprochene Häufung auf bestimmte Tage nicht constatiren, der Beginn des Fiebers vertheilt sich vielmehr seheinbar willkürlich auf die Tage der 1. und 2. Woche. Am häufigsten ist noch der 6. bis 9. Tag befallen, also das Ende der 1. und der Anfang der 2. Woche, die zusammen 15 Fälle $=60$ pCt. aufweisen, während der 4 . und 5. Tag nur schwach besetzt sind. Es erkrankten nämlich

$$
\begin{gathered}
\text { am Tag } \quad 3=11 \text { pCt. } \\
\text { 5. } " \quad 1=4 \% \\
" 6 . " 5=20 "
\end{gathered}
$$


Baisch, Der Einfluss der Soheidendesinfection auf die Morbidität etc.

$$
\begin{gathered}
\text { am } 7 \text { Tag } 5=20 \text { pCt. } \\
" \text { 8. } " 2=8 n \\
n 9 . " 3=11 "
\end{gathered}
$$

Bei den Nichtgespülten finden. wir somit die prognostisch günstigeren Spätfieber überwiegen. Wenn wir noch hinzufügen, dass die durchschnittliche Dauer der Erkrankung bei den mehr als einen Tag Fiebernden, bei Gespülten wie Nichtgespülten, 3 Tage währte, und dass die Höhe der Temperatur hier wie dort im Mittel 39,4 ${ }^{\circ}$ betrug, so erhellt, dass auch nach diesen Gesichtspunkten die Nichtdesinficirten sich nicht schlechter stellten als die Desinficirten.

Ist somit kein Zweifel möglich, dass die Scheidendesinfection mit Sublimat, statt die Zahl der Fieberfälle zu verringern, sie im Gegentheil vermehrt, so ist doch die Ursache dieser nachtheiligen Wirkung auf den Verlauf des Wochenbetts aus der klinischen Beobachtung nicht ohne Weiteres ersichtlich. Eher werden wir von der bakteriologischen Untersuchung Aufklärung erwarten dürfen. Die Spülungen richten sich ja doch in erster Linie gegen etwaige Streptokokken in der Vagina. Ihr Nutzen musste sich also in einem Rückgang der Streptokokkeninfectionen manifestiren, und wir würden einen gewissen Vortheil der Desinfection auch dann zugeben können, wenn zwar quantitativ eine Steigerung der Zahl der Erkrankungen nach der Spülung constatirt werden müsste, es sich aber bei genauerer Untersuchung herausstellen würde, dass diese Infectionen qualitativ harmlos sind, insofern sie nicht auf Streptokokkeninfection beruhen.

Bei den Nichtgespülten fanden sich in den Uteruslochien:

A) der 11 Kranken

Streptokokken

Staphylokokken

Mischinfection

Strepto- und Staphylokokken

u. bact. coli

Bact. coli

Gonokokken

Nicht näher bestimmte Keime 1 ,

Keine Keime
B) der 15 Eintagsfieber

$\begin{array}{ll}3 \text { mal } & 3 \mathrm{mal} \\ 2 n & 1 \%\end{array}$


Bei den Gespülten:

A) der 18 Kranken

Streptokokken

Staphylokokken

Mischinfection
$11 \mathrm{mal}$

$1 \pi$

3

(mit Eihautretention)

Gonokokken

Nicht näher bestimmte Keime

Keine Keime
1

0

$2 n$
B) der 32 Eintagsfieber

$$
\begin{aligned}
& 8 \mathrm{mal} \\
& 4 n \\
& 3 n
\end{aligned}
$$

(1 mal Eihautretention)

$3 \mathrm{mal}$

$4 n$

10

Die sicher erwiesenen Streptokokkeninfectionen betragen demnach:

$$
\begin{aligned}
& \text { bei den Gespülten } 38 \text { pCt. } \\
& \text { bei den Nichtgespülten nur } 23 "
\end{aligned}
$$

Die Scheidenspülungen sind somit nicht im Stande, gerade die gefürchtesten Keime, die specifischen Puerperalfiebererreger, zu vernichten.

Dieses Versagen der Sublimatscheidenspülung gegenüber den Streptokokken lässt sich nun auch durch bakteriologische Untersuchung der Scheidenlochien vor und nach der Geburt erweisen.

Schon vor Jahren hat Bumm darauf aufmerksam gemacht, dass durch die Spülungen ein Florawechsel der Vagina begünstigt werde, sofern die harmlosen Scheidenbacillen verschwinden und statt ihrer Kokken auftreten. Auch Menge sieht in dieser Entfernung oder Vernichtung der gegenüber den Streptokokken antagonistisch bedeutungsvollen Pilze eine wesentliche Ursache der Schädlichkeit der Scheidendesinfection.

Ich habe bei 20 Schwangeren das Scheidensekret einige Tage vor und dann wieder in den ersten 2 Tagen nach der Geburt untersucht und bin zu Ergebnissen gekommen, die die Angaben Bumm's voll bestätigen und die Befürchtung von Menge wohlbegründet erscheinen lassen. Von den Untersuchten wurden 9 während der Geburt gespült, 11 nicht.

Bei den 11 Nichtgespülten, deren Scheidensekret vor der Geburt entweder nur. Stäbchen - oder überwiegend Stäbchen mit wenig Kokken enthielt, liessen sich nach der Geburt einmal im Ausstrichpräparat keine Keime nachweisen, in der Cultur dagegen gingen in der Bouillon wie bei aerober und anaerober Züchtung auf 
Agar Stäbchen und Streptokokken auf. In 5 Fällen waren im Ausstrichpräparat nur Stäbchen, in 5 weiteren neben Stäbchen auch Kokken vorhanden, erstere jedoch in überwiegender Menge, Kokken allein fanden sich niemals.

Der Charakter des Sekrets war also kurz nach der Geburt derselbe wie vorher: es enthielt entweder nur harmlose Keime oder wenigstens diese in der Uebermacht.

Bei den 9 Gespülten dagegen, deren Scheidensekret ebenso wie das der Nichtgespülten vor der Geburt bei der einen Hälfte nur Stäbchen, bei der anderen überwiegend Stäbchen mit wenig Kokken enthalten hatte, war nach der Geburt 4 mal das $\Lambda$ usstrichpräparat frei von Keimen. In den Culturen wuchsen in diesen 4 Fällen 2 mal nur Streptokokken, 2 mal neben diesen auch Stäbchen. In 3 Fällen fanden sich Stäbchen und Kokken in annähernd gleicher Vertheilung und" 2 mal fanden sich nur Kokken.

Der Keimgehalt des Sekrets war, also nicht sehr stark, aber doch deutlich in dem Sinn geändert, dass die harmlosen Bacillen zurückgedrängt, z. Th. ganz entfernt, z. Th. von den Kokken überwuchert waren. Die Kokken erwiesen sich in allen Fällen bei weiterer Züchtung als aerob wie anaerob wachsende typische Streptokokken.

Diese Befunde zeigen, dass es der Scheidendesinfection nicht gelingt, Keime von der Art der Erreger des Puerperalfiebers aus der Vagina zu entfernen, dass vielmehr ihre Ansiedlung oder Vermehrung dadurch nur begünstigt wird auf Kosten der harmlosen, ungefährlichen, ja direct nützlichen Stäbchenformen. Diese gegen jede Aenderung ihrer Lebensbedingungen ausserordentlich empfindlichen Keime fallen dem Sublimat in erster Linie zum Opfer, während die resistenteren Kokken davon nicht getroffen werden.

Was ergiebt sich nun aus diesen für die Frage der Zweckmässigkeit der Scheidendesinfection so eindeutigen Resultaten für das Problem der Selbstinfection?

Da wir durch den Gebrauch von Gummibandschuhen die Händeinfection mit grösster Sicherheit eliminirt haben, da ferner die überhaupt nicht Untersuchten in derselben Häufigkeit erkrankten wie die 20 und $30 \mathrm{mal}$ innerlich Untersuchten, so scheinen die 7,6 pCt. Fieberfälle, die wir bei Spontangeburten nach zuverlässiger Abscheidung der accidentellen Erkrankungen noch zu verzeichnen 
haben, kaum anders denn als Selbstinfectionen, als Infectionen mit präexistirenden Scheidenkeimen gedeutet werden za können.

Analysiren wir aber die einzelnen Fälle genauer, so muss diese Deutung wesentliche Einschränkungen erfahren.

Aus der Gesammtsumme der 76 genitalen Fieberfälle lassen sich zunächst als besondere Groppe diejenigen Fälle abscheiden, wo das Zurückbleiben grösserer Eihautreste Veranlassung zu saprischer Infection wurde. Die bakteriologische Untersuchung der Uteruslochien stellte in diesen Fällen das Vorhandensein einer Mischinfection mit Bact. coli, Staphylokokken und Streptokokken fest. Für diese prognostisch günstigen und therapeutisch dankbaren Infectionen ist das Zustandekommen durch spontane Ascendenz von Vulva- und Scheidenkeimen entlang dem todten Material unbestritten. Rechnen wir zu dieser Gruppe auch die Fälle, in denen Bact. coli oder nicht näher zu bestimmende meist obligat anaerobe Keime in den Uteruslochien gefunden wurden, so repräsentiren sie mit 16 Fällen 1,6\% aller unserer Wöchnerinnen und $20 \%$ oder ein Füftel der Fiebernden.

Eine zweite, wohl characterisirte Gruppe stellen die gonorrhoischen Endometritiden dar. Obwohl hier von einer Selbstinfection im eigentlichen Sinne des Wortes gesprochen werden kann, sind die Fälle doch von den septischen Infectioner scharf abzutrennen. Olshausen will sie bekanntlich überhaupt nicht zu Puerperalfieber gerechnet wissen. Die Höhe des Antheils der Gonorrhoe an der Wochenbettsmorbidität wird in verschiedenen Kliniken verschieden sein, in den vorwiegend ländlichen Kreisen, aus denen sich unsere Schwangeren resultiren, spielt sie eine untergeordnete Rolle. Es erkrankten von sämmtlichen Wöchnerinnen nur $0,6 \%$, von sämmtlichen Fiebernden stellt dies $8 \%$ dar. Es handelt sich in allen 6 Fällen um leichte Erkrankungen im Spätwochenbett.

In 21 Fällen $=2,1 \%$ der Wöchnerinnen und $28 \%$ der Erkrankten fanden sich keine Keime in den Uteruslochien. Da sich auch sonst am Körper der Erkrankten keine Ursache des Fiebers nachweisen liess, haben wir auch diese Fälle, die übrigens sämmtlich sehr leicht verliefen, zu den Genitalerkrankungen gerechnet. Es ist aber keine Frage, dass bei der Leichtigkeit, mit der eine Streptokokken-Endometritis bakteriell nachzuweisen ist, auch diese Fälle nicht für die Möglichkeit einer durch Scheidenkeime spontan entstehenden puerperalen Sepsis verwerthet werden dürfen. 
342 Baisch, Der Einfluss der Scheidendesinfection auf die Morbidität etc.

Es bleiben nun noch 33 oder wenn wir ganz streng sein und auch die. Fälle von Mischinfection mitrechnen wollen, in denen sich neben anderen Keimen Streptokokken im Uterus fanden, 38 Streptokokken- und Staphylokokkeninfectionen, genau die Hälfte aller Fieberfälle, die wir bei unseren 1000 Wöchnerinnen beobachtet haben. Auf diese 38 Fälle hätte sich der Beweis für das Vorkommen und die klinische Bedeutung der Autoinfection mit septischen Keimen zu stützen.

Sehen wir sie uns darum genauer an.

Klinisch sind die Fälle zunächst dadurch charakterisirt, dass der erste Temperaturanstieg eintrat

$$
\begin{aligned}
& \text { innerhalb der ersten } 6 \text { Tage } 19 \mathrm{mal} \\
& \text { nach dem 6. Tag ebenfalls } 19 \mathrm{mal} .
\end{aligned}
$$

Es spricht ein hoher Grad von Wahrscheinlichkeit für die auch von Werth und Fehling für solche Spätfieber getheilte Annahme, dass in den letzteren 19 Fällen, die erst im Laufe der zweiten Woche nach der Geburt und zwar sämmtlich nur leicht und vorübergehend fieberten, die Bakterieninvasion erst im Wochenbett erfolgte.

Damit reduciren sich die Fälle von septischer Selbstinfection unter der Geburt auf 19 Erkrankungen. Und auch diese 19 Fälle rücken in eigenartige Beleuchtung, wenn wir sehen, dass 5 davon auf die Nichtgespülten, 14 auf die Gespülten entfallen.

Zunächst die 5 Erkrankungen der Nichtdesinficirten:

Nur 1 davon war eine typische Erkrankung, sofern sie am 4. Tag post part. einsetzte. Das Fieber $39^{\circ}$ dauerte 2 Tage.

Eine 2. Erkrankung war ein Eintagsfieber am 6 Tag.

Die andern 3 Erkrankungen waren mehrtägige Fieberfälle, die merkwürdigerweise schon am 1. (1 Fall) und 2. Tag mit hohem Fieber und erheblichen subjectiven Klagen einsetzten. Eine dieser Wöchnerinnen war erst 5 Tage vor der Geburt in die Klinik eingetreten, eine zweite verheimlichte zunächst die Geburt und meldete sich erst mit bereits geborenem Kinde bei der Oberhebamme.

Es ist ausserordentlich wahrscheinlich, wenn auch naturgemäss ein stricter Beweis dafür nicht erbracht werden kann, dass es sich in diesen Fällen nicht um Spontan- und Autoinfectionen gehandelt hat, sondern dass auch hier eine Infection von aussen im Spiel ge- 
wesen ist, über deren Natur wir freilich nur Vermuthungen hegen können.

Dass gegenüber den 5 Streptokokken-Endometritiden der 500 Nichtdesinficirten bei den Gespülten 14 Fälle, also die dreifache Zahl, beobachtet wurden, scheint mir bei der grossen Zahl der verglichenen Wöchnerinnen kein Zufall zu sein.

Von diesen 14 Fällen waren nur 5 leichte, eintägige Störungen am 4. (3 Fälle), 5. und 6. Tag (je ein Fall), 9 mal handelte es sich um mehrtägige Erkrankungen.

In diesen 9 Fällen setzte das Fieber am 4., 5. und 6. Tag ein (je 3 Fälle). Diese typisehen Beginne weisen wohl mit Sicherheit auf Infectionen unter der Geburt hin.

Die Scheidendesinfection hat diese 9 ernsteren Erkrankungen nicht verhindert, ja es drängt sich ohne Weiteres der Gedanke auf, dass durch die Spülung selbst und während ihrer Ausführung die Infection durch Einimpfung von Vaginalstreptokokken in die lädirten, in ihrer Vitalität herabgesetzten Gewebe direct veranlasst wurde. Die Scheidendesinfection hat, statt die Selbstinfection zu beschränken nnd aufzuheben, sie offenbar geradezu provocirt. Es ist dies freilich keine Selbstinfection im eigentlichsten Sinne des Wortes mehr, keine spontane, unvermeidbare Erkrankung, sondern eine verhütbare und arteficielle.

So schrumpfen bei näherer Betrachtung die Fälle von echter Selbstinfection auf ein Minimum zusammen. Es bleibt von einer eingehenden bakteriologisch-klinischen Analyse jedes einzelnen Falles streng genommen nur der einzige Fall von Erkrankung einer Nichtgespülten übrig, die am 4. Tag post part. zweitägiges Fieber bekam!

Aber auch wenn wir noch die 3 atypischen Erkrankungen mit heranzieben wollten, in denen wir eine anderweitige Infectionsquelle nur vermuthen, nicht beweisen können - es ist bei 1000 Wöchnerinnen nur noch eine verschwindend kleine Zahl. Bei Berücksichtigung der in allen Anstalten gegebenen Möglichkeit einer uncontrolirbaren Infection durch die Schwangere selbst oder andere, nicht geburtshilfliche Personen, und des weiteren Umstandes, dass auch bei peinlichster Aufmerksamkeit eine Keimverschleppung nicht absolut als ausgeschlossen angesehen werden darf, kommt dieses Ergebniss thatsächlich einer Negation der Selbstinfection gleich. 
344 Baisch, Der Einfluss der Scheidendesinfection auf die Morbidität etc.

Giebt es keine Selbstinfection, so scheint es nur logisch, von jedem. Versuch, die Scheidenkeime zu vernichten, von vornherein Abstand zu nehmen. Und doch lehrt uns eben die bakteriologische Untersuchung der Uteruslochien, dass das echte "Puerperalfieber". in allen Fällen einem Eindringen von pathogenen Keimen in die Uterushöhle seinen Ursprung verdankt. Unsere Erfahrungen, dass bei Abrechnung der accidentellen Erkrankungen 7,6 pCt. aller spontan Niedergekommenen fieberhaft erkranken, zeigen unzweifelhaft, dass die Prophylaxe des Puerperalfiebers noch keineswegs zu den erledigten Problemen der Geburtshilfe gehört. Diese Prophylaxe kann, da uns die Gummihandschuhe volle Sicherheit gegen Keimeinschleppung geben, nur darin bestehen, dass wir anderweitig eingedrungene oder präexistirend vorhapdene Keime an der Ascendenz in die Uterushöhle verhindern. Die Sublimatspülungen sind dazu nicht im Stande, da sie zu sehr die physiologischen Schutzkräfte der Vagina alteriren. Aber die von Döderlein mit Milchsäureeingiessungen gemachten Erfahrungen beweisen, dass der Versuch, in der Scheide vorhandene pathogene Keime zu vernichten, nicht von vornherein als aussichtsios $\mathrm{zu}$ betrachten ist. Auf einen zweiten Weg hat neuerdings $\mathrm{Z}$ weifel hingewiesen: er will durch Entfernung des Blutes aus der Scheide kurz nach der Geburt den pathogenen Keimen das Nährmaterial entziehen, ihre Ascendenz verhindern.

Da wir uns keineswegs mit der Thatsache trösten, dass die Erkrankungsgefahr bei Spontangeburten nur $7-8$ pCt. betrage und eine tödtliche Erkrankung so gut wie ausgeschlossen sei, es vielmehr als eine der wichtigsten Aufgaben der Geburtshilfe betrachten, die Puerperalfieberfälle noch weiter herabzusetzen und jeden dahingehenden Vorschlag willkommen heissen, so haben wir nunmehr eine neue Versuchsreihe begonnen. Die Sublimatdesinfection der Scheide vor der Geburt allerdings haben wir aufgegeben. Unsere mit der Zuverlässigkeit eines wissenschaftlichen Experiments angestellten Untersuchungen baben uns die Ueberzeugung verschafft, dass sie nur schädlich sind. An ihrer Stelle haben wir das $Z$ weifelsche Verfahren gesetzt und unterziehen ihm abwechselnd jede zweite Wöchnerin. Sobald diese Versuche die entsprechende Zahl von Beobachtungen aufzuweisen haben, soll über den Erfolg berichtet werden. 


\section{Lit e ratur.}

Ahlfeld, Beiträge zur Lehre vom Resorptionsfieber in der Geburt und im Wochenbett und von der Selbstinfection. Zeitschr. f. Geburtsh. u. Gyn. Bd. 27. S. 466.

Ablfeld, Beiträge zur Frage von der Entstehung der feberhaften Wochenbettserkrankungen. Zeitschr. f. Geburtsh. u. Gyn. Bd. 43̈. S. 191.

Baumm, Beiträge zur Puerperalfeberfrage. Asepsis und Antisepsis in der Geburtshülffe. Dieses Archiv. Bd. 52.

Bumm, Zur Frage der inneren Desinfection Kreissender. Centralbl. f. Gynäk. 1892. No. 9. S. 153.

Bumm, Zur Kenntniss des Eintagsfiebers im Wochenbett. Centralbl, f. Gyn. 1897. No. 45 .

Bumm, Ueber Aetiologie und Pathogenese des Kindbettfiebers. Verhandl. d. deutschèn Ges. f. Gyn. Bd. VIII. S. 276.

Bretschneider, Klinische Versuche über den Einfluss der Scheidenspülungen währeend der Geburt auf den Wochenbettsverlauf. Dieses Arch, Bd. 63 . S. 453.

Döderlein, Zur Verhütung der Infection Gebärender. Berliner klin. Wochenschr. 1898. No. 50.

Döderlein-Günther, Zur Desinfection des Geburtscanals. Dieses Archiv. Bd. 34. H. 1. S. 111.

Döderlein, Ueber das Verhalten pathogener Keime zur Scheide. Deutsche med. Wochenschr. 1895. No. 10.

Döderlein, Ueber das Vorkommen von Spaltpilzen in den Lochien des Uterus und der Vagina gesunder und kranker Wöchnerinnen. Habilit.-Schrift. Leipzig. 1887.

Dödorlein, Das Scheidensekret und seine Bedeutung für das Puerperalfieber. Leipzig. 1892.

Döderlein, Zur Entstehung und Verhütung des Puerperalfiebers. Verhandl. d. deutschen Gesellsch. f. Gynäk. Bd. X. S. 563.

Döderlein-Winternitz, Bakteriologie der puerperalen Sekrete. Hegar's Beiträge. 1900. Bd. II. H. 2.

Fehling, Klinische Untersuchungen über Morbidität im Kindbett. Verhandl. d. deutschen Ges. f. Gyn. Bd. VIII. S. 321.

Frommel, Zur Prophylaxe der Wochenbetterkrankungen. Deutsche med. Wochenschr. 1892. No. 10. S. 202.

Hofmeier, Zur Verhütung des Kindbettfiebers. Münchener med. Wochenschr. 1900. No. 37 und 1902. No. 18 u. 19.

Hofmeier, Die Verhütung puerperaler Infectionen. Die deutsche Klinik. Bd. IX. S. 33.

Krönig, Klinische Versuche über den Einfluss der Scheidenspülungen während der Geburt auf den Wochenbettsverlauf. Münchener med. Wochenschr. 1900. No. 1.

Leopold u. Goldberg, Ueber die Entbehrlichkeit der Scheidenausspülung bei regelmässigen Geburten. Dieses Arch. 1891. Bd. 40. S. 439. 
346 Baisch, Der Einfluss der Scheidendesinfection auf die Morbidität ete.

Menge, Antisepsis und Asepsis bei der Geburt. v. Winckel's Handbuch der Geburtshülfe. Bd. I. 2. Hälfte. S. 1173.

Olshausen, Ueber den Begriff des Puerperalfiebers. Centralbl. f. Gyn. 1899. No. 1.

Olshà usen, Ueber Antisepsis und Asepsis in der Gynäkologie und Geburtshülfe. Berliner klin. Wochenschr. 1899. No. 45.

Schuhmacher, Bạkteriologisch-klinische Studien zur Händedesinfection. Dieses Archiv. Bd. 68. S. 399.

Steffeck, Uebèr Desinfection des weiblichen Genitalcanals. Zeitschr. f. Geb. 1888. Bd. XV. S. 395.

Steffeck, Bakteriologische Begrändung der Selbstinfootion. Zeitschr. f. Geburtsh. 1890. Bd. XX. S. 339.

Sticher, Händesterilisation und Wochenbettsmorbidität. Zeitschr. f. Geburtshülfe. Bd. 45. H. 3.

Stolz, Studien zur Bakteriologie des Genitalcanals, Graz. 1903.

Walthard, Bakteriologische Untersuchungen des weiblichen Genitalsekrets in graviditate und im Puerperium. Dieses Arch. Bd. 48. H, 2. 